\title{
La composition en acides aminés de quelques fromages courants ${ }^{*}$
}

\author{
par \\ A. DE VUYST, W. VERVACK, M. VANBELLE \\ et M. FOULON
}

\section{INTRODUCTION}

Le lait et ses nombreux dérivés, parmi lesquels les fromages de tous types, entrent pour une part de plus en plus grande dans l'alimentation humaine. Ils sont une source très intéressante de protéines alimentaires de haute qualité.

L'utilisation digestive de ces protéines est de l'ordre de 95 p. 100 à 98 p. 100 .

La teneur en protéine du lait est de l'ordre de 3 p. 100 en moyenne, celle des fromages varie entre 9,10 p. 100 pour les fromages blancs et 29,10 p. 100 pour un fromage à pâte dure cuite, du type Gruyère.

Le passage du lait au fromage implique toute une série de traitements industriels, allant de la coagulation du lait à l'affinage en caves en passant par l'égouttage du caillé, son découpage, moulage, salage, etc.

De plus, certains fromages (Gruyère par exemple) subissent au cours de la fabrication, une cuisson supplémentaire d'une durée de $45 \mathrm{mn}$ à $50 \mathrm{mn}$ à la température de $55^{\circ} \mathrm{C}$.

Tous ces traitements peuvent être la cause de modifications plus ou moins profondes de la composition chimique des constituants du lait.

Dans le présent travail, nous étudierons tout spécialement les transformations subies par les protéines du lait et des acides aminés qui les composent. Nous étudierons aussi l'influence de ces traitements sur le blocage de la lysine et donc sur sa disponibilité (available lysine, selon Carpenter) [1, 2].

\footnotetext{
* Ce travail a été réalisé au Centre de Recherches Zootechniques de l'Université de Louvain (Dir. Prof A. DE VUYST) 3042 Lovenjoel (Belgique), avec des subsides de I'I.B.A.N. (Institut Belge pour l'Alimentation et la Nutrition).
} 


\section{MATERIEL ET METHODES}

\section{1) Matériel} ment :

Différents types de produits laitiers ont été étudiés et notam-

Yoghourts

Fromages blancs

Fromages de Hollande

Fromage Port-Salut

Fromage de Chester

Fromage de Tilsitt

Fromage de Gruyère

Fromage de Brie

Fromage Camembert

Fromage de Herve

Fromage de Bruxelles

Fromages fondus
18 échantillons

19 échantillons

6 échantillons

8 échantillons

8 échantillons

4 échantillons

6 échantillons

6 échantillons

7 échantillons

3 échantillons

3 échantillons

16 échantillons.

\section{2) Méthodes}

Les méthodes analytiques suivies sont :

a) détermination de la matière azotée totale, selon Kjeldahl,

b) détermination de la matière sèche, selon normes F.I.L. [3],

c) détermination des acides aminés selon Spackman, Stein et Moore [4] sur Amino Acid Analyzer Unichrom (Beckman),

d) détermination de la lysine disponible selon la méthode de Rexen et Christensen modifiée par nous [5].

Un assez grand nombre d'échantillons de chaque type de fromages sont analysés, de manière à pouvoir établir une moyenne statistiquement valable, accompagnée de l'écart type [6]. Nous pouvons ainsi voir s'il y a des différences significatives ou non entre les différents types de fromages analysés.

\section{RESULTATS EXPERIMENTAUX}

Les résultats de nos analyses sont consignés dans les tableaux suivants. Nous y renseignons la composition en acides aminés du lait, du yoghourt et des différents fromages analysés.

Les compositions en acides aminés sont données en grammes d'acides aminés pour $100 \mathrm{~g}$ de matière azotée totale.

Les teneurs en matière azotée totale et en matière sèche sont également renseignées. 


\section{DISCUSSION}

Les traitements thermiques (stérilisation, évaporation, etc.) des produits laitiers ont une action néfaste sur la qualité et la valeur biologique de ces produits, car certains acides aminés, et principalement la lysine, sont assez sensibles à pareils traitements thermiques.

L'histidine est également assez sensible à ces traitements thermiques, de même que l'arginine.

Dans un précédent article, nous avons étudié le problème de la lysine disponible dans les produits laitiers [2] et nous avons pu déterminer l'action des traitements industriels sur la disponibilité de cet acide aminé essentiel.

\section{Yoghourt}

Le yoghourt est préparé à partir de lait pasteurisé à $84^{\circ} \mathrm{C}-85^{\circ} \mathrm{C}$ pendant quelques secondes. Ce traitement rapide n'a pratiquement aucune influence sur la composition en acides aminés et sur la disponibilité de la lysine.

La teneur en lysine totale du yoghourt est légèrement inférieure à celle de la lysine totale du lait frais, et est identique à celle du lait pasteurisé. La lysine disponible représente 93 p. 100 de la lysine totale.

Au point de vue des acides aminés essentiels, nous constatons une diminution des teneurs en thréonine, isoleucine et arginine, ce qui donnera au yoghourt une valeur biologique légèrement plus basse que celle du lait.

\section{Fromage blanc}

La fabrication du fromage blanc se fait au départ de lait entier, de lait écrémé ou de lait battu. On n'emploie que du lait pasteurisé et l'emprésurage se fait à une température de $18^{\circ} \mathrm{C}$ à $22^{\circ} \mathrm{C}$.

C'est un fromage obtenu avec un minimum de manipulation et qui doit se manger peu de temps après la fabrication.

On constate, par rapport au lait, une diminution des teneurs en thréonine et en isoleucine.

La lysine totale diminue également par rapport au lait.

La disponibilité de la lysine n'est plus que 82 p. 100, alors que pour le lait elle était de 97 p. 100 environ.

Cette diminution des teneurs en trois acides aminés essentiels entraînera une perte de valeur biologique de la protéine du fromage blanc, par rapport à la protéine du lait. 


\section{TABLEAU 1}

Composition en acides aminés du lait, du yoghourt et du fromage blanc en grammes d'acides aminés pour $100 \mathrm{~g}$ de matière azotée totale

\begin{tabular}{|c|c|c|c|c|}
\hline Acides aminés & Lait & Yoghourt & Fromage & blanc \\
\hline $\begin{array}{l}\text { Acide aspartique } \\
\text { Thréonine } \\
\text { Sérine } \\
\text { Acide glutamique } \\
\text { Proline } \\
\text { Glycine } \\
\text { Alanine } \\
\text { Valine } \\
\text { Méthionine } \\
\text { Isoleucine } \\
\text { Leucine } \\
\text { Tyrosine } \\
\text { Phénylalanine } \\
\text { Lysine } \\
\text { Histidine } \\
\text { Arginine } \\
\text { Lysine disponible }\end{array}$ & $\begin{array}{rr}7,90 & \pm 0,26 \\
5,00 & 0,23 \\
6,20 & 0,26 \\
21,70 & 0,63 \\
10,10 & 0,21 \\
2,10 & 0,08 \\
3,60 & 0,04 \\
6,70 & 0,20 \\
3,40 & 0,20 \\
6,50 & 0,12 \\
9,90 & 0,25 \\
4,90 & 0,25 \\
5,04 & 0,22 \\
9,44 & 0,08 \\
3,12 & 0,04 \\
3,70 & 0,12 \\
9,19 & 0,12\end{array}$ & $\begin{array}{rr}8,10 & \pm 0,34 \\
4,20 & 0,17 \\
6,32 & 0,20 \\
19,99 & 0,90 \\
12,57 & 0,87 \\
2,47 & 0,18 \\
4,37 & 0,35 \\
8,44 & 0,51 \\
3,03 & 0,16 \\
5,30 & 0,14 \\
1,29 & 0,22 \\
5,15 & 0,15 \\
5,57 & 0,13 \\
9,25 & 0,13 \\
3,15 & 0,08 \\
3,45 & 0,09 \\
8,61 & 0,26\end{array}$ & $\begin{array}{r}7,06 \pm \\
3,65 \\
6,10 \\
20,84 \\
13,63 \\
2,35 \\
4,44 \\
8,16 \\
3,22 \\
4,96 \\
11,16 \\
5,65 \\
5,45 \\
8,68 \\
3,05 \\
3,68 \\
7,13\end{array}$ & $\begin{array}{l}0,36 \\
0,15 \\
0,17 \\
0,65 \\
0,73 \\
0,18 \\
0,33 \\
0,25 \\
0,15 \\
0,26 \\
0,35 \\
0,17 \\
0,22 \\
0,13 \\
0,07 \\
0,08 \\
0,33\end{array}$ \\
\hline $\begin{array}{l}\text { Protéines totales } \\
\text { Matière sèche }\end{array}$ & $\begin{array}{rr}3,31 & 0,10 \\
11,02 & 0,19\end{array}$ & $\begin{array}{rr}4,45 & 0,15 \\
11,88 & 0,48\end{array}$ & $\begin{array}{r}9,10 \\
18,27\end{array}$ & $\begin{array}{l}0,36 \\
0,84\end{array}$ \\
\hline $\begin{array}{l}\text { Nombre d'échantillons } \\
\text { analysés }\end{array}$ & 18 & 18 & 19 & \\
\hline
\end{tabular}

\section{Les fromages à pâte ferme}

Port-Salut : On ne constate que très peu de diminution des acides aminés essentiels et parfois on trouve des valeurs supérieures à celles du lait.

La valeur biologique de la protéine se trouvera donc très peu diminuée par rapport au lait.

Au point de vue lysine totale, il y a très peu de différence avec la teneur en lysine totale du lait.

La lysine disponible est assez fortement diminuée et n'est plus que de 7,62 p. 100 contre 9,19 p. 100 pour le lait.

Hollande : On note un abaissement des teneurs en thréonine et en arginine.

La perte en lysine totale est faible, de même que celle de la lysine disponible. Le taux de lysine disponible représente encore 94 p. 100 de la lysine totale. 
TABLEAU 2

Composition en acides aminés de fromages à pâte ferme en grammes d'acides aminés pour $100 \mathrm{~g}$ de matière azotée totale

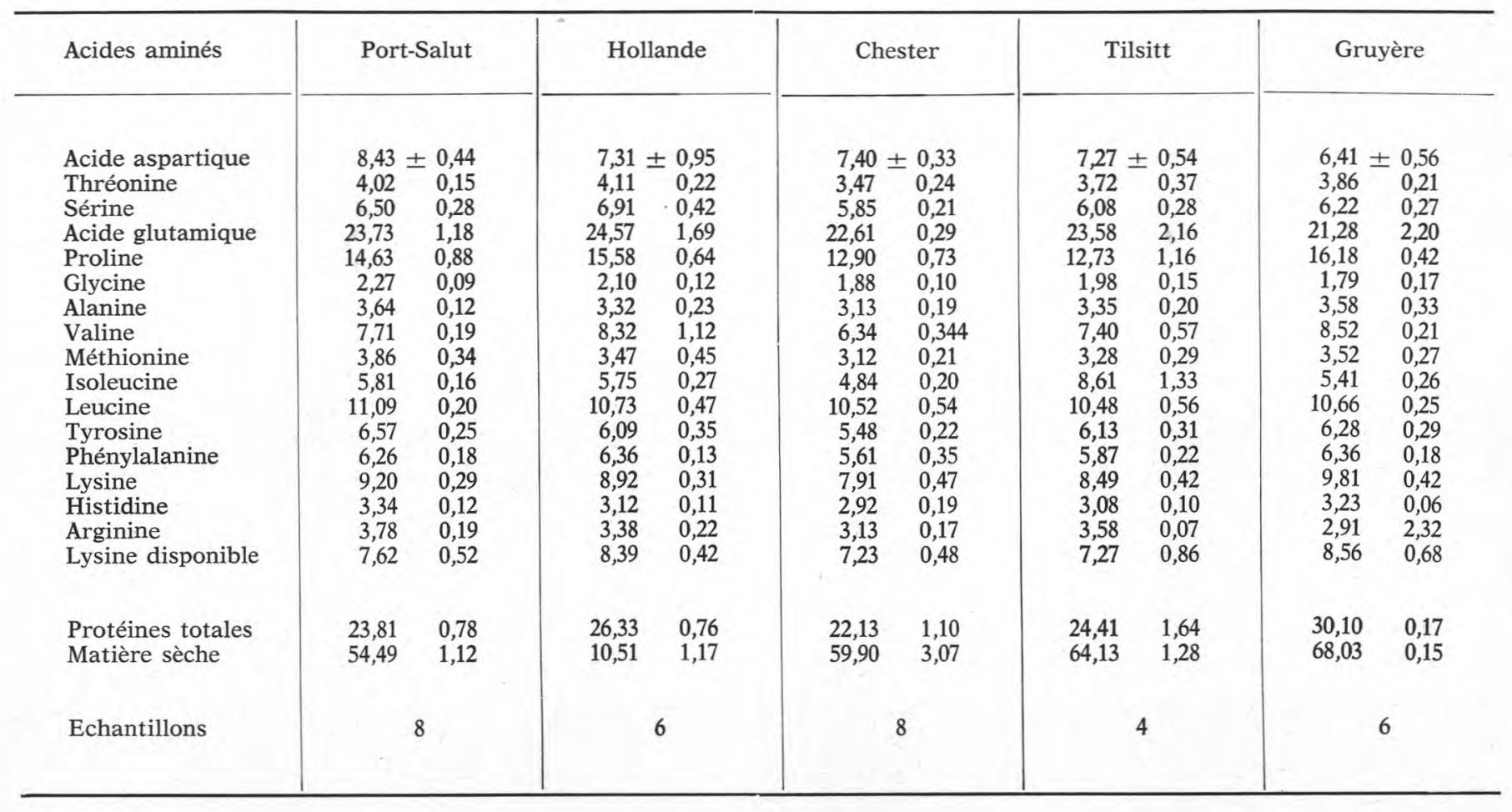


La valeur biologique de la protéine du fromage de Hollande est très intéressante et elle se rapproche très fort de celle du lait.

Chester : Ce type de fromage se rapproche très fort du fromage de Hollande au point de vue de la composition générale en acides aminés.

Il ne s'en différencie que par une teneur en lysine disponible plus faible : 7,23 p. 100 contre 8,39 p. 100 pour le fromage de Hollande.

La valeur biologique de sa protéine sera de ce fait légèrement diminuée.

Tilsitt : Au point de vue composition en acides aminés, il se rapproche très fort du fromage de Chester.

Sa valeur biologique lui est sensiblement égale, mais on constate une légère diminution de valeur biologique par rapport au fromage de Hollande et au lait.

Fromage de Gruyère: Appartient au groupe des fromages à pâte ferme ayant subi un traitement thermique assez prononcé, principalement destiné à assécher les grains de caillé et à supprimer certains germes thermolabiles.

La température de brassage est maintenue à $53^{\circ} \mathrm{C}-57^{\circ} \mathrm{C}$ durant $45 \mathrm{mn}$ à $60 \mathrm{mn}$.

On constate une répercussion de ce fait sur la diminution de la disponibilité de la lysine, qui ne représente plus que 87 p. 100 de la lysine totale.

On ne constate par contre qu'une très faible diminution de la lysine totale.

\section{Les fromages à pâte fermentée}

Les fromages de ce type appartiennent au groupe des fromages qui subissent un affinage et une fermentation plus ou moins longue : Camembert, Brie, Herve et fromage de Bruxelles.

Camembert : Les pertes en acides aminés sont plus élevées que pour les autres fromages. On constate une teneur en lysine totale de 8,56 p. 100 alors que pour le lait on constatait une teneur de 9,44 p. 100.

La teneur en lysine disponible est de 7,73 p. 100 alors que pour le lait elle est de 9,19 p. 100.

Plusieurs autres acides aminés diminuent également et notamment : thréonine, valine, isoleucine et leucine.

Ce fait diminue la valeur biologique de la protéine du Camembert, mais cette valeur biologique reste néanmoins encore intéressante.

L'augmentation de la teneur en ammoniaque est l'indice d'une dégradation plus grande de la protéine : ainsi, dans le Camembert, on retrouve de 21 p. 100 à 27 p. 100 d'azote sous forme d'azote ammo- 
TABLEAU 3

Composition en acides aminés de fromages à pâte fermentée en grammes d'acides aminés pour $100 \mathrm{~g}$ de matière azotée totale

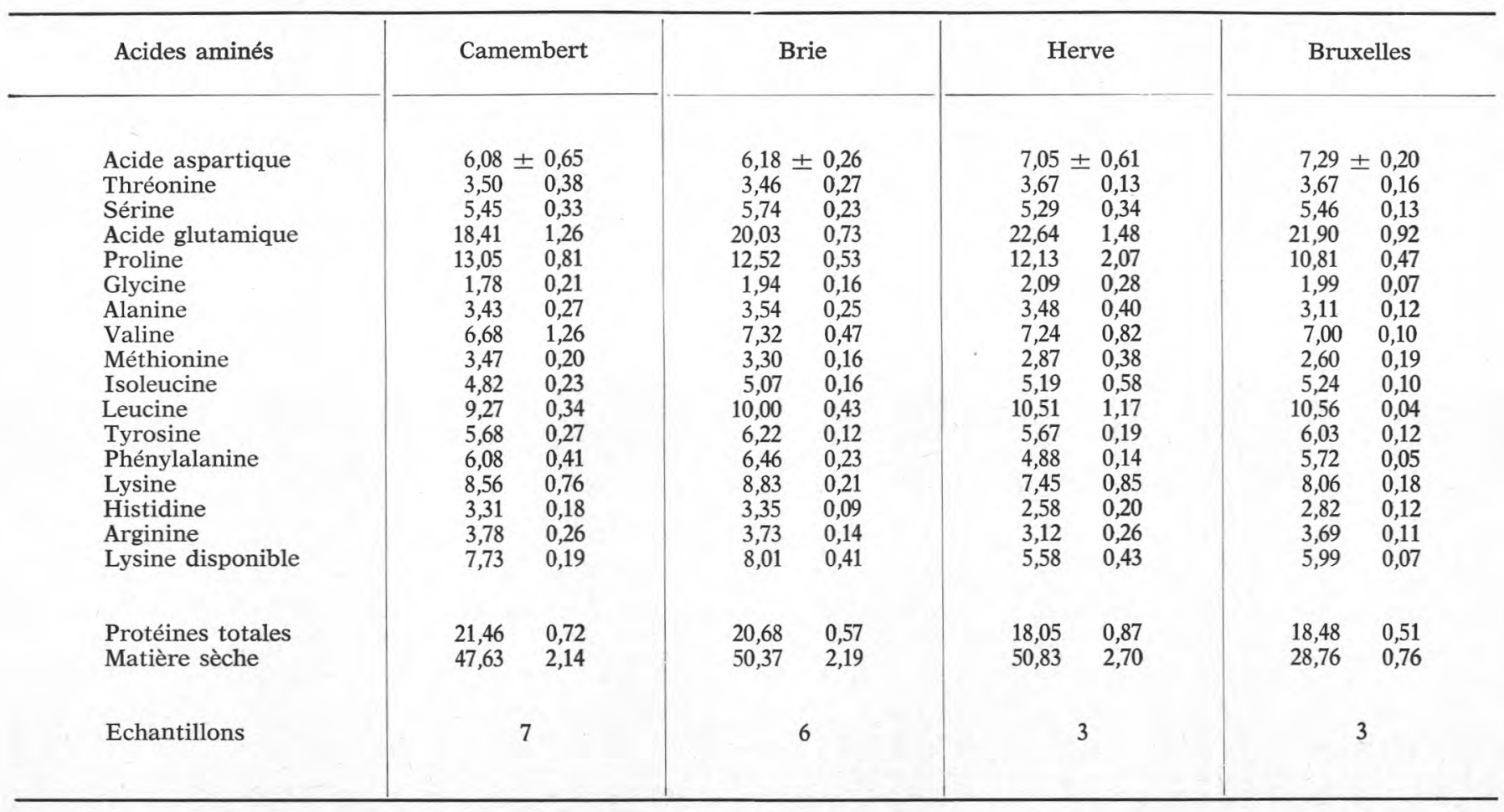


niacal, alors que dans le fromage de Hollande, cette teneur est de 10,5 p. 100 à 14,5 p. 100 .

Brie : Au point de vue composition en acides aminés, le Brie est très comparable au Camembert. Il ne s'en distingue que par une teneur plus élevée en acide glutamique.

Les teneurs en lysine totale et en lysine disponible sont très sensiblement les mêmes.

Ces deux types de fromages auront donc une valeur biologique très semblable.

Fromage de Herve : Ressemble assez bien au Camembert et au Brie au point de vue composition brute en acides aminés, sauf pour les trois acides aminés basiques (lysine, histidine et arginine) où l'on observe des teneurs plus faibles.

La teneur en lysine totale tombe à 7,45 p. 100 .

On observe aussi une diminution de la teneur en lysine disponible qui tombe au taux de 5,58 p. 100 .

La valeur biologique de la protéine du fromage de Herve sera donc plus faible que celle des autres fromages à pâte fermentée.

Fromage de Bruxelles: Ne se différencie pratiquement pas des trois autres fromages à pâte fermentée, au point de vue composition brute en acides aminés.

La teneur en lysine totale et en lysine disponible est très semblable à celle du fromage de Herve.

La valeur biologique de la protéine du fromage de Bruxelles sera semblable à celle du fromage de Herve, mais plus faible que celles du Camembert et du Brie.

\section{Les fromages fondus}

Les fromages fondus sont composés d'autres fromages maturés et affinés, moulus et broyés et auxquels on ajoute des sels de fonte et de l'eau.

On peut y adjoindre toutes sortes d'ingrédients tels que : jambon, salami, crevettes, champignons, etc.

Ils peuvent être préparés à partir de fromages de premier choix ou de fromages présentant des défauts physiques, mais pas des défauts chimiques. La cuisson dure de 8 à $12 \mathrm{mn}$ à une température oscillant entre $75^{\circ} \mathrm{C}$ et $85^{\circ} \mathrm{C}$.

Au point de vue acides aminés, on constate à nouveau une diminution des teneurs en thréonine, isoleucine et arginine. 
Les pertes en lysine totale sont très minimes ; la lysine disponible représente encore 91,78 p. 100 de la lysine totale.

La perte de valeur biologique par rapport au lait sera donc assez faible.

\section{TABLEAU 4}

Composition en acides aminés des fromages fondus en grammes d'acides aminés pour $100 \mathrm{~g}$ de matière azotée totale

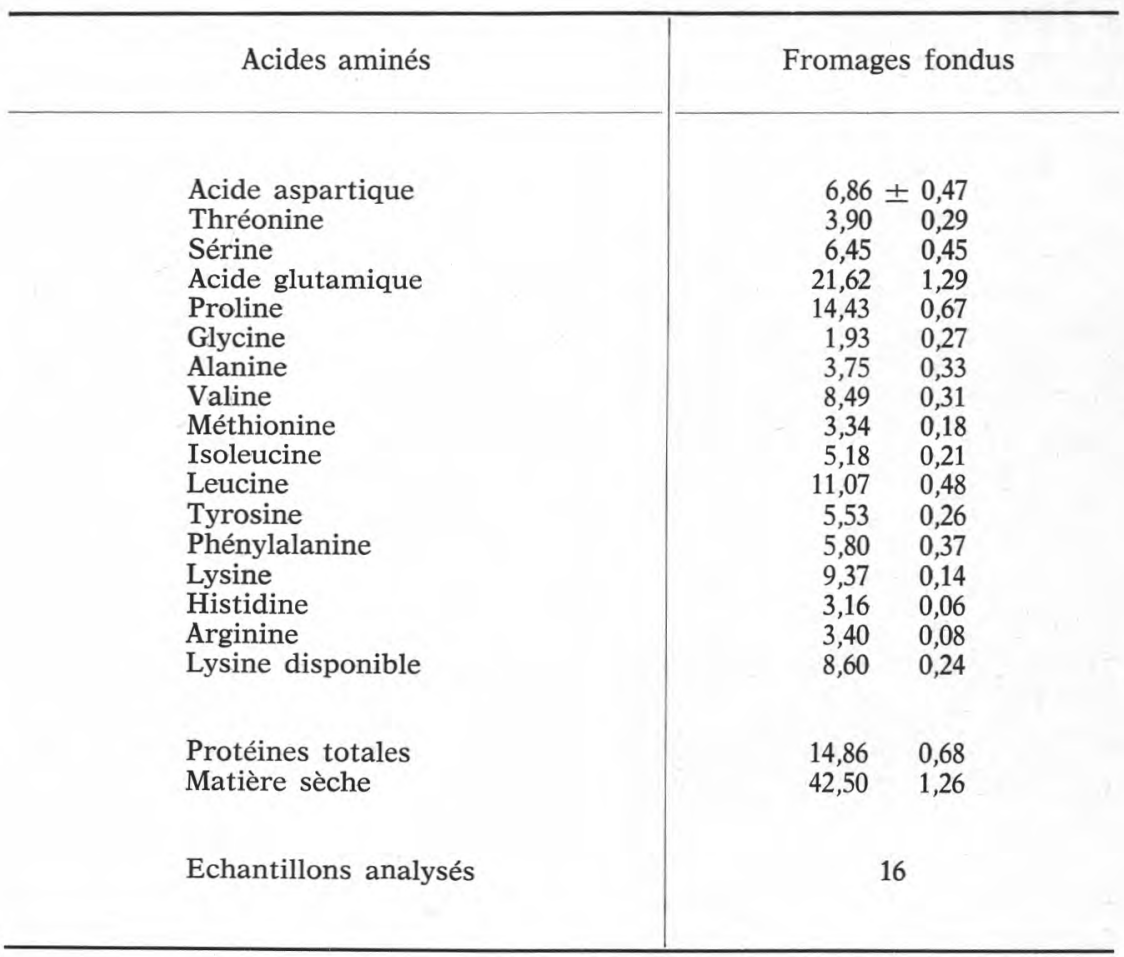

\section{La valeur biologique calculée de la protéine des fromages}

La valeur biologique calculée de la protéine de ces différents fromages (ou Index d'Acides Aminés Essentiels I.A.A.E.) en prenant le lait comme base de référence (index I.A.A.E. du lait $=100$ ), ou index de Nehring, nous donne une idée des différences existant entre les divers types de fromages analysés : 
Index de Nehring des différents fromages analysés

\begin{tabular}{l|c}
\hline \multicolumn{1}{c|}{ Fromages } & Index de Nehring \\
\cline { 2 - 2 } & \\
Référence lait & 100 \\
Yoghourt & 96 \\
Fromage blanc & 94 \\
Port-Salut & 97 \\
Hollande & 97 \\
Chester & 97 \\
Tilsitt & 96 \\
Gruyère & 94 \\
Camembert & 93 \\
Brie & 94 \\
Herve & 91 \\
Bruxelles & 95 \\
Fromages fondus & 95 \\
& \\
\hline
\end{tabular}

On voit que la fabrication des différents fromages et du yoghourt n'a pas entamé fortement la valeur biologique de la protéine du lait.

Ils oscillent entre 91 et 97.

Le yoghourt qui est un lait fermenté a un index de Nehring de 96 qui prouve que la valeur biologique de la protéine du lait n'a pas souffert du traitement industriel.

Le fromage blanc n'a qu'un index de 94, quoiqu'il ne subisse qu'un traitement industriel minimum : emprésurage et caillage, suivi d'égouttage.

Les quatre fromages à pâte ferme ont un index de Nehring compris entre 96 et 97 .

Le Gruyère, pour sa part, a un index de Nehring de 94, plus faible que les autres fromages à pâte ferme. Cette baisse de valeur biologique doit être attribuée à la cuisson inhérente à la préparation de ce genre de fromage. Les fromages à pâte fermentée, Brie et Camembert, ont des index de Nehring très proches de 93 et 94 . Les index de Nehring des fromages de Bruxelles et de Herve sont assez différents l'un de l'autre.

Enfin, les fromages fondus ont un index moyen de 95.

\section{R és u m é}

Dans le présent travail, les auteurs ont étudié la séquence des acides aminés composant la protéine de différents types de fromages et dérivés du lait, tels que yoghourt, fromage blanc, fromages à 
pâte ferme, fromages à pâte ferme et cuite, fromages à pâte fermentée et fromages fondus.

Sur la base de la composition en acides aminés essentiels, ils calculent l'index de valeur biologique de Nehring, par comparaison avec les acides aminés composant la protéine du lait.

Ils arrivent à la conclusion que la fabrication des différents types de fromages n'altère que très peu la valeur biologique de la protéine du lait. La teneur en lysine disponible n'est que très peu affectée ce qui semble indiquer que les réactions de Maillard ne jouent pas un rôle très important dans le cas du traitement de la protéine du lait pour donner les différents fromages étudiés.

\section{S u m m a r y}

In this work, the authors have studied the amino acids composition of various cheese proteins and of yohourt. They studied among others curd cheese, cheeses of the dutch cheese type, gruyere cheese, fermented cheeses and melted cheese of various type.

On the basis of the amino acid composition they calculate the Nehring index, an estimation of the biological value of the protein of cheese, by comparison with the amino acids composition of milk protein.

They concluded that biological value of milk protein and of cheese protein are quite similar.

The availability of lysine (following the conception of Carpenter) is very little affected during the preparation of the various types of cheeses. It is an indication that the Maillard reaction between lysine and sugars are not intensive during the cheese preparation.

\section{Bibliographie}

[1] CARpenter (K. J.) and Ellinger (1955). - The determination of available lysine in feedingstuffs. Biochemical Journal, vol. 61, XI.

[2] De Vuyst (A.), Vervack (W.), Charlier (H.) et Jadin (V.) (1972). - La teneur en lysine totale et disponible du lait, des fromages et des produits de boulangerie ayant subit un traitement industriel. Le Lait (Revue générale des questions laitières), tome LII, $\mathrm{n}^{\circ} 517$, juillet-août, 444-453.

[3] Fédération Internationale de Laiterie (International Dairy Federation), normes d'analyses. Bruxelles, square Vergote.

[4] Spackman (D. H.), Stein (W. H.) and Moore (S.) (1958). - Automatic recording apparatus for use in the chromatography of amino acids. Analytical Chemistry, vol. $30, \mathrm{n}^{\circ} 7,1190-1206$.

[5] ReXen (B.) and Christensen (B.) (1967). - The determination of available lysine in feedingstuffs. Automation in Analytical Chemistry. Technicon International Ardsley Chauncey, New-York.

[6] SNEDECOR (1950). - Statistical Methods. Ames Ioxa The Ioxa State College Press Fourth Printing. 